\title{
Serum NGAL, BNP, PTH, and albumin do not improve glomerular filtration rate estimating formulas in children
}

\author{
Julie Mouron-Hryciuk ${ }^{1} \cdot$ François Cachat ${ }^{1} \cdot$ Paloma Parvex $^{2} \cdot$ Thomas Perneger $^{3} \cdot$ Hassib Chehade $^{1}$
}

Received: 18 August 2020 / Revised: 2 March 2021 / Accepted: 4 March 2021 / Published online: 10 March 2021

(C) The Author(s) 2021

\begin{abstract}
Glomerular filtration rate (GFR) is difficult to measure, and estimating formulas are notorious for lacking precision. This study aims to assess if the inclusion of additional biomarkers improves the performance of eGFR formulas. A hundred and sixteen children with renal diseases were enrolled. Data for age, weight, height, inulin clearance (iGFR), serum creatinine, cystatin C, neutrophil gelatinase-associated lipocalin (NGAL), parathyroid hormone (PTH), albumin, and brain natriuretic peptide (BNP) were collected. These variables were added to the revised and combined (serum creatinine and cystatin C) Schwartz formulas, and the quadratic and combined quadratic formulas. We calculated the adjusted $r$-square $\left(r^{2}\right)$ in relation to iGFR and tested the improvement in variance explained by means of the likelihood ratio test. The combined Schwartz and the combined quadratic formulas yielded best results with an $r^{2}$ of 0.676 and 0.730 , respectively. The addition of BNP and PTH to the combined Schwartz and quadratic formulas improved the variance slightly. NGAL and albumin failed to improve the prediction of GFR further. These study results also confirm that the addition of cystatin $\mathrm{C}$ improves the performance of estimating GFR formulas, in particular the Schwartz formula.

Conclusion: The addition of serum NGAL, BNP, PTH, and albumin to the combined Schwartz and quadratic formulas for estimating GFR did not improve GFR prediction in our population.
\end{abstract}

\section{What is Known:}

- Estimating glomerular filtration rate (GFR) formulas include serum creatinine and/or cystatin C but lack precision when compared to measured GFR.

- The serum concentrations of some biological parameters such as neutrophil gelatinase-associated lipocalin (NGAL), parathyroid hormone (PTH),

albumin, and brain natriuretic peptide (BNP) vary with the level of renal function.

What is New:

- The addition of BNP and PTH to the combined quadratic formula improved its performance only slightly. NGAL and albumin failed to improve the prediction of GFR further.

Keywords Glomerular filtration rate $\cdot$ Neutrophil gelatinase-associated lipocalin $\cdot$ Brain natriuretic peptide $\cdot$ Parathyroid hormone $\cdot$ Albumin $\cdot$ Child

Communicated by Gregorio Paolo Milani

Hassib Chehade

Hassib.chehade@chuv.ch

Julie Mouron-Hryciuk

julie_hryciuk@hotmail.com

François Cachat

Francois.Cachat@chuv.ch

Paloma Parvex

Paloma.Parvex@hcuge.ch
Thomas Perneger

Thomas.Perneger@hcuge.ch

1 Women-Mother-Child Department, Pediatric Nephrology Division, Lausanne University Hospital and University of Lausanne, Rue du Bugnon 46, 1011 Lausanne, Switzerland

2 Department of Pediatrics, Geneva University Hospital and University of Geneva, Geneva, Switzerland

3 Division of Clinical Epidemiology, Faculty of Medicine, University of Geneva, Geneva, Switzerland 


\section{Introduction}

Estimation of glomerular filtration rate (eGFR) is important in clinical practice [1]. Several formulas have been developed for this purpose; however, all these formulas lack precision, and much effort is actually made in order to improve eGFR prediction. Serum creatinine (Scr) is the most commonly used endogenous marker to estimate GFR but with relative imprecision owing to variation of non-GFR determinants of Scr, e.g., muscle mass, high meat-containing diet, or tubular secretion. Moreover, an increase in Scr may not be observed until a substantial decrease of at least 40\% in GFR has occurred [2]. Recently, serum cystatin C (Scyst C) has emerged as an alternative to or a complement of creatinine measurement in the evaluation of kidney function. Several studies have shown a better estimation of GFR using combined Scr and Scyst C measurements [3, 4]. However, Scyst C can be influenced by uncontrolled thyroid disease or immunosuppressive therapies [5, 6], also leading to a decrease in eGFR precision. Until now, measurement of inulin clearance represents the gold standard method to assess GFR. Other exogenous markers (i.e., Cr-51 EDTA, iohexol) have been used as well; however, all these methods can be cumbersome and are not always available in clinical practice [7].

To overcome the above-mentioned limitations, many serum biomarkers have been incorporated in mathematical models in order to improve bedside formula for GFR estimation [8, 9]. The Kidney Disease Outcomes Quality Initiative (K/DOQI) guidelines recommend estimating GFR in adults and children using Scr and/or Scyst $\mathrm{C}$ based predictive equations [1]. The most widely used formula is the Schwartz formula, which was developed in 1976 and revised in 2009 [10-12]. We have also developed a Scr and a combined (Scr and Scyst C) quadratic formula in order to better assess GFR in Swiss children [13, 14]. However, all these new formulas lack precision when compared to measured GFR. Much effort is invested in finding new biomarkers to improve the accuracy of bedside GFR estimation. This study aimed to evaluate if the addition of serum neutrophil gelatinase-associated lipocalin (NGAL), brain natriuretic peptide (BNP), parathyroid hormone (PTH), and albumin improves the performance of the Schwartz and the quadratic formulas, compared to urinary inulin clearances.

The choice of biomarkers to be included in this study was based on several considerations. NGAL has recently emerged as a predictive biomarker in chronic kidney disease (CKD) [15]. PTH is a recognized biomarker in CKD. Fu et al. [16] showed a significant negative correlation between PTH and eGFR. In addition, Okamoto $\mathrm{K}$ et al. [17] demonstrated that changes in PTH levels were associated with kidney function and renal outcome. Regarding albumin, patients with CKD often present with decreased appetite secondary to uremia and consequently may develop malnutrition $[9,18]$. Finally, BNP has been used in CKD progression and GFR estimation in adult patients $[19,20]$.
To the best of our knowledge, there are no adult nor pediatric studies evaluating the impact of BNP, PTH, NGAL, and albumin in estimating GFR.

\section{Patients and methods}

\section{Patients}

Children with chronic kidney diseases (CKD) who were referred to our clinic for GFR measurement between August 2012 and June 2013 were retrospectively included. Children were aged between 3 and 18 years. Causes of CKD included congenital and acquired single kidney, obstructive or reflux uropathy, polycystic kidney disease, and other various diagnoses (Bartter syndrome, patients with history of hemolytic uremic syndrome, post-chemotherapy). Patients who were unable to void spontaneously were excluded from the study.

\section{Measurements and analytic methods}

GFR was measured using the gold standard method - urinary inulin clearance (iGFR). Inulin was measured using an AutoAnalyzer 3 system, as previously reported by our research group $[13,14]$. At the time of measuring patient's GFR, additional data were collected: height $(\mathrm{cm})$, weight $(\mathrm{kg})$, Scyst $\mathrm{C}$ (mg/l), Scr (mg/dl), serum blood urea nitrogen (BUN) in mmol/l, serum NGAL ( $\mathrm{ng} / \mathrm{ml})$, intact serum PTH $(\mathrm{pg} / \mathrm{ml})$, serum BNP $(\mathrm{pg} / \mathrm{ml})$, and serum albumin $(\mathrm{mg} / \mathrm{l})$. Scyst $C$ was measured using the particle-enhanced nephelometric immunoassay (Siemens Healthcare Diagnostics). Scr was analyzed using the kinetic colorimetric compensated Jaffe method (Roche Diagnostics, cobas 8000) which was calibrated against the enzymatic method and standardized against the reference isotope dilution mass spectrometry method. Serum BUN was measured with the enzymatic assay (Roche Diagnostics, cobas 8000), and PTH was measured with enzyme immunoassay (Immulite $2000 \mathrm{XPi} / \mathrm{Siemens),} \mathrm{BNP}$ with electro-chemiluminescence immunoassay (Roche Diagnostics, cobas 8000/e801), and serum albumin with bromocresol green (Roche Diagnostics, cobas 8000 ). The eGFR was calculated with the following equations: revised Schwartz, combined Schwartz, quadratic, and combined quadratic formulas (Table 1).

\section{Statistical analysis}

Continuous data are presented as median with interquartile range $[\mathrm{IQR}]$ and categorical as percentages. We used a simple linear regression to examine association of continuous variables (age, sex, height, serum cystatin C, creatinine, NGAL, BNP, PTH, and albumin) with inulin clearance. We first obtained the variance in inulin clearance explained by the basic formulas, i.e., the coefficient determination ( $r$-squared). Then we added to the best 
Table 1 Equations used for the estimation of glomerular filtration rate

\begin{tabular}{ll}
\hline Revised Schwartz formula & $0.413 \times(\mathrm{Ht} / \mathrm{Scr})$ \\
Combined Schwartz formula & $39.8 \times(\mathrm{Ht} / \mathrm{Scr})^{0.456} \times(1.8 / \mathrm{Scys})^{0.418} \times(30 / \mathrm{BUN})^{0.079} \times(\mathrm{Ht} / 1.4)^{0.179}[\times 1.076$ if female $]$ \\
Quadratic formula & $60 \times(\mathrm{Ht} / \mathrm{Scr})-6.25 \times(\mathrm{Ht} / \mathrm{Scr})^{2}+0.48 \times$ age $-[25.68$ if female or 21.53 if male $]$ \\
Combined quadratic formula & $0.42 \times(\mathrm{Ht} / \mathrm{Scr})-0.04 \times(\mathrm{Ht} / \mathrm{Scr})^{2}-14.5 \times \mathrm{Scys}+0.69 \times$ age $+[18.25$ if female or 21.88 if male $]$
\end{tabular}

Glomerular filtration rate expressed in $\mathrm{ml} / \mathrm{min} / 1.73 \mathrm{~m}^{2}$

$\mathrm{Ht}$, height expressed in cm; Scr, serum creatinine expressed in mg/dl; Scys, serum cystatin c expressed in mg/l; BUN, blood urea nitrogen expressed in $\mathrm{mg} / \mathrm{dl}$; age expressed in years

fitted linear regression each of the additional variables (serum cystatin $\mathrm{C}$, NGAL, BNP, PTH, and serum albumin), one at a time, obtained the adjusted $r$-square of the models, and tested the improvement in variance explained by means of the likelihood ratio test. Significance was set at $p<0.05$.

We attempted to construct a parsimonious multivariate model predicting inulin clearance including all statistically significant predictors.

\section{Results}

A total of hundred and sixteen patients were enrolled in this study. Patients' median [IQR] age was 12.6 [8.4-15.5] years, and $52 \%$ were boys. Children's demographic characteristics are summarized in Table 2. Ninety-one percent of the patients had CKD stage I and II, and 9\% had a CKD stage III to V. All patients' data were well documented and recorded, without any missing data.

Median [IQR] iGFR was 82.5 [71.0-98.8] ml/min/1.73 $\mathrm{m}^{2}$. The median values for eGFR using the revised and combined Schwartz formula, the quadratic, and the combined quadratic formula were $89.5,91.3,91.5$, and $88.0 \mathrm{ml} / \mathrm{min} / 1.73 \mathrm{~m}^{2}$, respectively. Results confirmed that the best empirical model for inulin clearance $\left(r^{2}=0.75\right)$ was the association of sex, inverse creatinine, inverse creatinine squared, height squared, and inverse cystatin $C$.

The combined quadratic formula performed best, with the highest $r^{2}$ value at 0.730 (Table 3 ) compared to other formulas $\left(r^{2}\right.$ at $0.569,0.676$, and 0.708 for the Schwartz, combined Schwartz, and quadratic formulas, respectively).

The addition of cystatin $\mathrm{C}$ to the Schwartz and the quadratic formulas improved $r^{2}$ the most (from 0.569 and 0.708 to 0.700 and 0.734 , respectively). Adding BNP and PTH also improved the variance of the combined quadratic formula slightly (from 0.708 to 0.738 for both, $p=0.042$ and 0.046 , respectively) (Table 3 ). The addition of NGAL and serum albumin did not increase $r^{2}$ (Table 3).

After adding BNP and PTH to the combined Schwartz formula, the $r^{2}$ changed from 0.676 to 0.694 and 0.673 , respectively. Regarding the addition of NGAL and serum albumin the combined Schwartz formula, we observed a change of $r^{2}$ from 0.676 to 0.673 and 0.682 , respectively. In neither case were we able to specify a multivariate model that was significantly better than the basic formulas plus cystatin $\mathrm{C}$.

\section{Discussion}

In this study, we found that the addition of serum NGAL, BNP, PTH, and albumin to the combined Schwartz and quadratic formulas for estimating GFR did not improve GFR prediction in our population. This study represents a new step in developing better GRF estimation equations with the addition of several renal biomarkers. We attempted to evaluate the usefulness of adding BNP, PTH, NGAL, and albumin in the estimation of GFR. BNP is a peptide hormone whose

Table 2 Overview of the patient characteristics

\begin{tabular}{ll}
\hline Number of patients & 116 \\
Height in cm & $151[129-166]$ \\
Weight in kg & $42.9[27.2-57.6]$ \\
BMI & $18[16-21]$ \\
Renal disease & \\
Congenital and acquired single kidney & $20(17)$ \\
Obstructive or reflux uropathy & $53(46)$ \\
Polycystic kidney disease & $19(16)$ \\
Miscellaneous & $24(21)$ \\
Inulin clearance (ml/min/1.73m $\left.{ }^{2}\right)$ & $82.5[71.0-92.8]$ \\
Estimated GFR (ml/min/1.73m $\left.{ }^{2}\right)$ & \\
Revised Schwartz formula & $89.5[72.0-99.0]$ \\
Combined Schwartz formula & $91.3[82.1-101.4]$ \\
Quadratic formula & $91.5[78.2-98.0]$ \\
Combined Quadratic formula & $88.0[79.2-93.0]$ \\
CKD stages & \\
Stage l & $36(31)$ \\
Stage ll & $70(61)$ \\
Stage iii & $4(3)$ \\
Stage lV and V & $6(5)$ \\
\hline
\end{tabular}

Continuous variables are presented as median with IQR and categorical as $n(\%)$

$I Q R$ interquartile range, $B M I$ body mass index expressed in $\mathrm{kg} / \mathrm{m}^{2}$. Miscellaneous includes the following diagnoses: Bartter syndrome, patients with history of hemolytic uremic syndrome, and postchemotherapy 
Table 3 Ability to explain variance in iGFR of existing eGFR formulas (adjusted rsquare), and increase after the inclusion of additional biomarkers in a linear regression model

\begin{tabular}{lllll}
\hline & Revised Schwartz & Combined Schwartz & Quadratic & Combined quadratic \\
\hline Basic formula & 0.569 & 0.676 & 0.708 & 0.730 \\
+ NGAL & 0.566 & 0.673 & 0.708 & 0.731 \\
+ BNP & 0.620 & 0.694 & 0.728 & 0.738 \\
+ PTH & 0.651 & 0.673 & 0.728 & 0.738 \\
+ Albumin & 0.608 & 0.682 & 0.717 & 0.731 \\
\hline
\end{tabular}

$N G A L$ neutrophil gelatinase-associated lipocalin, $B N P$ brain natriuretic peptide, $P T H$ parathyroid hormone physiological role is regulation of intravascular blood volume and vascular tone. Increase of BNP level may reflect renal failure. Wiley et al. demonstrated in a cohort of 1739 adult patients a statistically significant correlation between BNP and eGFR using the MDRD formula [19]. Takase et al. showed similar results in a population of 282 patients [20]. We found a positive effect of adding BNP to the combined quadratic formula; however, the effect remains extremely small, albeit significant. Regarding PTH, it is well known that PTH increases with CKD progression. Several studies analyzed the correlation between PTH and GFR. This correlation may depend on the degree of CKD [21-23]. Similar to BNP, this study showed that adding PTH led to a slight increase in the performance of the combined Scr and Scyst $\mathrm{C}$ formulas. However, the clinical impact is limited, creating a more complicated formula. In a recent study, there was a significant correlation between serum NGAL and measured GFR in children with CKD. The authors concluded that serum NGAL may prove useful in the quantitation of CKD, especially at low levels of measured GFR, where, by correlation analysis, NGAL outperformed cystatin C [15]. In addition, Bolignano et al. [24] demonstrated that NGAL is an independent predictor of CKD progression. Despite the fact that NGAL has been shown to be a good diagnostic and prognostic biomarker of acute and chronic renal failure $[15,25,26]$, the addition of NGAL did not lead to a better prediction of measured GFR. Finally, albumin has been previously used in adult GFR estimation formulas [9]. Levey A et al. showed that for every $10 \%$ of albumin change, there was a $3.1 \%$ change in GFR [9]. This study is the first one to include albumin in eGFR pediatric formulas. Similar to NGAL, albumin did not increase the performance of combined creatinine and cystatin C GFR estimation formulas. In the same vein, other researchers attempted to develop formulas incorporating new biomarkers such as $\beta 2$ Microglobulin or $\beta$-Trace Proteins, with conflicting results $[27,28]$. Recently, Inker LA et al. nicely demonstrated that the incorporation of $\beta 2$-Microglobulin and $\beta$-Trace Protein into adult prediction formulas improved their performance, without incorporating the race data [29]. Chen $\mathrm{N}$ et al. also showed the beneficial addition of $\beta 2$-Microglobulin and $\beta$ Trace Protein in improving GFR estimation in a Chinese population, however without significant clinical impact [30]. The utility of these two biomarkers in predicting GFR should be investigated further.

Our study results confirm that the addition of Scyst C improves the performance of estimating GFR formulas, in particular the Schwartz formula. Recently, den Bakker et al. demonstrated in a large cohort of 408 patients that Scr and Scyst C were the most powerful predictors of GFR, even in the absence of height data [31]. Deng et al. confirmed a better accuracy and applicability of the multivariate eGFR equations including Scr and Scyst C compared to univariate formulas [32].

The strength of this study is the use of inulin clearance. All variables were measured simultaneously during inulin test, therefore reducing potential variation in their level due to progression of renal failure with time. In addition, to the best of our knowledge, this is the first study investigating the potential role of NGAL, BNP, PTH, and albumin in GFR estimation in a pediatric population.

However, the study results and conclusions are limited by the fact that we have included only Swiss children, and by a small number of patients with $\mathrm{iGFR}<60 \mathrm{ml} / \mathrm{min} / 1.73 \mathrm{~m}^{2}$. These limit the generalizability of the results to nonCaucasian children and to those with moderate to severe CKD.

In conclusion, serum creatinine and cystatin $\mathrm{C}$ remain the best predictors for GFR estimation. The addition of other parameters (i.e., BNP and PTH) may be beneficial but should balance the complexity of the new equations and its associated cost.

Abbreviations BNP, Brain natriuretic peptide; BUN, Serum blood urea nitrogen; CKD, Chronic kidney disease; GFR, Glomerular filtration rate; iGFR, Urinary inulin clearance; K/DOQI, Kidney Disease Outcomes Quality Initiative; NGAL, Serum neutrophil gelatinase-associated lipocalin; PTH, Parathyroid hormone; Scr, Serum creatinine; Scyst C, Serum cystatin $\mathrm{C}$

Availability of data and material The datasets generated during and/or analyzed during the current study are available from the corresponding author.

Authors' contributions Conceptualization: $\mathrm{HC}$ and FC, Data curation: JMH, FC, HC, TP, and PP. Formal analysis: TP. Roles/writing-original draft: JMH, FC, HC, TP, and PP. Writing-review and editing: JMH, FC, $\mathrm{HC}, \mathrm{TP}$, and PP. 
Funding Open Access funding provided by Universite de Lausanne. The study has been supported by the pediatric nephrology laboratory of Lausanne University Hospital.

\section{Declarations}

Ethics approval The study has been approved by the local Ethic Committee (Lausanne Ethic Research Committee Protocol number $345 / 12)$.

Consent to participate and for publication The study has been approved by the Research Ethic Committee of Lausanne.

Competing interests The authors declare no competing interests.

Open Access This article is licensed under a Creative Commons Attribution 4.0 International License, which permits use, sharing, adaptation, distribution and reproduction in any medium or format, as long as you give appropriate credit to the original author(s) and the source, provide a link to the Creative Commons licence, and indicate if changes were made. The images or other third party material in this article are included in the article's Creative Commons licence, unless indicated otherwise in a credit line to the material. If material is not included in the article's Creative Commons licence and your intended use is not permitted by statutory regulation or exceeds the permitted use, you will need to obtain permission directly from the copyright holder. To view a copy of this licence, visit http://creativecommons.org/licenses/by/4.0/.

\section{References}

1. National Kidney Foundation (2002) K/DOQI clinical practice guidelines for chronic kidney disease: evaluation, classification, and stratification. Am J Kidney Dis 39(2 Suppl 1):S1-S266

2. Prévot A, Martini S, Guignard J-P (2002) Glomerular filtration markers in pediatrics. Rev Med Suisse Romande 122(12):625-630

3. Fan L, Inker LA, Rossert J, Froissart M, Rossing P, Mauer M, Levey AS (2014) Glomerular filtration rate estimation using cystatin $\mathrm{C}$ alone or combined with creatinine as a confirmatory test. Nephrol Dial Transplant 29:1195-1203

4. Stevens LA, Coresh J, Schmid CH, Feldman HI, Froissart M, Kusek J, Rossert J, van Lente F, Bruce RD III, Zhang Y(L), Greene T, Levey AS (2008) Estimating GFR using serum cystatin $\mathrm{C}$ alone and in combination with serum creatinine: a pooled analysis of 3,418 individuals with CKD. Am J Kidney Dis 51(3):395406

5. Andersen TB, Eskild-Jensen A, Frøkiaer J et al (2009) Measuring glomerular filtration rate in children; can cystatin $\mathrm{C}$ replace established methods? A review. Pediatr Nephrol 24:929-941

6. Roos JF, Doust J, Tett SE, Kirkpatrick CMJ (2007) Diagnostic accuracy of cystatin $\mathrm{C}$ compared to serum creatinine for the estimation of renal dysfunction in adults and children-a meta-analysis. Clin Biochem 40:383-391

7. Santos J, Martins LS (2015) Estimating glomerular filtration rate in kidney transplantation: Still searching for the best marker. World J Nephrol 4:345-353

8. Cockcroft DW, Gault MH (1976) Prediction of creatinine clearance from serum creatinine. Nephron 16:31-41

9. Levey AS, Bosch JP, Lewis JB, Greene T, Rogers N, Roth D (1999) A more accurate method to estimate glomerular filtration rate from serum creatinine: a new prediction equation.
Modification of diet in renal disease study group. Ann Intern Med 130:461-470

10. Schwartz GJ, Brion LP, Spitzer A (1987) The use of plasma creatinine concentration for estimating glomerular filtration rate in infants, children, and adolescents. Pediatr Clin N Am 34:571-590

11. Schwartz GJ, Muñoz A, Schneider MF, Mak RH, Kaskel F, Warady BA, Furth SL (2009) New equations to estimate GFR in children with CKD. J Am Soc Nephrol 20:629-637

12. Schwartz GJ, Schneider MF, Maier PS, Moxey-Mims M, Dharnidharka VR, Warady B et al (2012) Improved equations estimating GFR in children with chronic kidney disease using an immunonephelometric determination of cystatin C. Kidney Int 82:445-453

13. Gao A, Cachat F, Faouzi M, Bardy D, Mosig D, Meyrat B-J, Girardin E, Chehade H (2013) Comparison of the glomerular filtration rate in children by the new revised Schwartz formula and a new generalized formula. Kidney Int 83:524-530

14. Chehade H, Cachat F, Jannot A-S, Meyrat B-J, Mosig D, Bardy D, Parvex P, Girardin E (2014) New combined serum creatinine and cystatin C quadratic formula for GFR assessment in children. Clin J Am Soc Nephrol 9:54-63

15. Mitsnefes MM, Kathman TS, Mishra J, Kartal J, Khoury PR, Nickolas TL, Barasch J, Devarajan P (2007) Serum neutrophil gelatinase-associated lipocalin as a marker of renal function in children with chronic kidney disease. Pediatr Nephrol 22:101-108

16. Fu S, Yu H, Li Y, Lv F, Deng J, Zhang F, Luan F, Zhao Y, Yao Y (2020) Multiple measures of mineral metabolism were associated with renal function in Chinese centenarians: a cross-sectional study. Front Med 7:120

17. Okamoto K, Fujii H, Goto S, Kono K, Watanabe K, Nishi S (2020) Changes in the whole/intact parathyroid hormone ratio and their clinical implications in patients with chronic kidney disease. $\mathrm{J}$ Nephrol 33:795-802

18. Cano N (2000) Malnutrition and chronic renal failure. Ann Med Interne (Paris) 151:563-574

19. Wiley CL, Switzer SP, Berg RL, Glurich I, Dart RA (2010) Association of B-type natriuretic peptide levels with estimated glomerular filtration rate and congestive heart failure. Clin Med Res 8: $7-12$

20. Takase H, Dohi Y (2014) Kidney function crucially affects B-type natriuretic peptide (BNP), N-terminal proBNP and their relationship. Eur J Clin Investig 44:303-308

21. Yuen NK, Ananthakrishnan S, Campbell MJ (2016) Hyperparathyroidism of renal disease. Perm J 20:15-127

22. Choi S-W, Kweon S-S, Lee Y-H, Ryu S-Y, Choi J-S, Nam H-S et al (2018) Parathyroid hormone levels are independently associated with eGFR and albuminuria: the Dong-gu study. J Nutr Sci Vitaminol 64:18-25

23. Tassone F, Gianotti L, Baffoni C, Pellegrino M, Castellano E, Borretta G (2015) KDIGO categories of glomerular filtration rate and parathyroid hormone secretion in primary hyperparathyroidism. Endocr Pract 21:629-633

24. Bolignano D, Lacquaniti A, Coppolino G, Donato V, Campo S, Fazio MR et al (2009) Neutrophil gelatinase-associated lipocalin (NGAL) and progression of chronic kidney disease. Clin J Am Soc Nephrol 337-344

25. Haase M, Bellomo R, Devarajan P, Schlattmann P, Haase-Fielitz A, NGAL Meta-analysis Investigator Group (2009) Accuracy of neutrophil gelatinase-associated lipocalin (NGAL) in diagnosis and prognosis in acute kidney injury: a systematic review and metaanalysis. Am J Kidney Dis 54:1012-1024

26. Krawczeski CD, Woo JG, Wang Y, Bennett MR, Ma Q, Devarajan P (2011) Neutrophil gelatinase-associated lipocalin concentrations predict development of acute kidney injury in neonates and children after cardiopulmonary bypass. J Pediatr 158:1009-1015.e1 
27. Witzel SH, Huang S-HS, Braam B, Filler G (2015) Estimation of GFR using $\beta$-trace protein in children. Clin J Am Soc Nephrol B10: 401-409

28. Scarr D, Bjornstad P, Lovblom LE, Lovshin JA, Boulet G, Lytvyn Y, Farooqi MA, Lai V, Orszag A, Weisman A, Keenan HA, Brent MH, Paul N, Bril V, Cherney DZI, Perkins BA (2019) Estimating GFR by serum creatinine, cystatin $C$, and $\beta 2$-microglobulin in older adults: results from the Canadian study of longevity in type 1 diabetes. Kidney Int Rep 4:786-796

29. Inker LA, Couture SJ, Tighiouart H, Abraham AG, Beck GJ, Feldman HI et al (2020) A new panel estimated GFR, including $\beta 2$-microglobulin and $\beta$-trace protein and not including race, developed in a diverse population. Am J Kidney Dis 4:S02726386(20)31126-4

30. Chen N, Shi H, Zhang L, Zuo L, Xie J, Xie D, Karger AB, Miao S, Ren H, Zhang W, Wang W, Pan Y, Minji W, Sui Z, Okparavero A,
Simon A, Chaudhari J, Eckfeldt JH, Inker LA, Levey AS (2020) GFR estimation using a panel of filtration markers in Shanghai and Beijing. Kidney Med 2:172-180

31. den Bakker E, Gemke R, van Wijk JAE, Hubeek I, Stoffel-Wagner B, Bökenkamp A (2018) Combining GFR estimates from cystatin $\mathrm{C}$ and creatinine-what is the optimal mix? Pediatr Nephrol 33: $1553-1563$

32. Deng F, Finer G, Haymond S, Brooks E, Langman CB (2015) Applicability of estimating glomerular filtration rate equations in pediatric patients: comparison with a measured glomerular filtration rate by iohexol clearance. Transl Res J Lab Clin Med 165:437-445

Publisher's note Springer Nature remains neutral with regard to jurisdictional claims in published maps and institutional affiliations. 